$\xi=-1$

\title{
Prebiological Organization of Molecules in Models and in the Matter of the Origin of Life
}

\author{
R.A. Zhogol \\ Candidate of medical Sciences, associate professor, Doctor of clinical laboratory diagnostics, Head of the laboratory of Polesskaya \\ Central district hospital in Kaliningrad region.
}

\begin{abstract}
The theoretical model of cell formation in cell selection is considered. The phenomenology of the process shows that molecular evolution did not have a Darwinian basis for development. According to the described model, cell formation is an inevitable process that develops a clear molecular mechanism. This mechanism is observed throughout the entire period of early development, beginning with the stage of initiation of the process, then at the stage of molecular complications and at the final final stage. The mechanism reveals the ideological concept according to which at an early stage there was a group of ribosome structures catalyzing reversible aminoacyltransfer, their activity led to the formation of a self-replicating cycle on a translational basis.
\end{abstract}

Keywords: The Origin Of Life; Molecular Evolution; A Replicator; Ribosomal Codons

\section{The Difficulties of Molecular Organization and the RNA World}

Every researcher who studies the issue of the origin of life should think about the question: what does he expect to learn, find out at the end of the path? Does he expect to see molecules that are still unknown to science with a "unique" structure or properties, or does the researcher support a realistic approach and believe that he will find unknown mechanisms at the end of the path, the totality of which will constitute some rules underlying the organization of molecules? This issue is extremely important, it forms the initial position in the consideration of the issue and therefore it must accompany our researcher throughout his long and difficult investigation

The organization of molecules is one of the greatest difficulties in the matter of the origin of life. All the efforts of various fields of science are aimed at revealing the reasons for organizing the molecular composition. Different directions in the studies give an understanding of the primary composition of the components, as well as the conditions for the existence of the first life forms. However, a complete and sufficiently detailed picture restoring the course of historical events of the organization of the primary molecular composition, regardless of the constituent components, remains unknown.

To find a suitable model capable of explaining the organization of molecules as a separate, independent phenomenon, it is necessary to divide the field of research into two separate topics:

1. Formation of a self-replicating cycle that can function on the basis of translation and expression of genes;

2. Determine the evolutionary conditions, due to which it becomes possible to transform the cycle towards its complication.

The search for a self-replicating cycle has always existed throughout the history of the issue of the origin of life. The selfreproducing cycle remains a subject of increased attention for theoretical constructions, it also serves the object of achieving the goal in experimental studies. Many researchers associate the problem of the origin of life with the formation of an effective replication system. Now, the focus is on RNA, with the search for molecules that will be able to catalyze their own replication. There is a popular hypothesis describing the early life form as an "RNA world" (Atkins et al., 2010). Efforts focus on finding the key components of RNA with RNA polymerase activity. Scientists are actively trying to get them artificially, through intelligent design with subsequent selection, as it is believed, conceptually reproducing artificial evolution. There is a wide variety of reactions catalyzed by ribozymes, so RNA can carry out aminoacylation, repair of RNA ruptures, nucleotide synthesis, formation of a peptide bond, and many other reactions. The study of ribozymes has long been transformed into an independent field of research (Doudna and Cech, 2002, Fedor and Williamson, 2005).

Despite active searches in the "RNA universe," no ribozymes have been found capable of replicating themselves. It is difficult to imagine how RNA molecules could combine catalytic properties with matrix molecules. If such molecules could still exist, then we would observe simple spontaneous formation of the same type of structures - in a certain sense spontaneous, uncontrolled, spontaneous phenomenon, leading to structural similarity of molecules, which would not facilitate understanding of molecular evolution. There are models in which the pathway to the effective ribozyme polymerase goes through intermediate stages (Briones C., Stitch M., Manrubia S.C. 2009). The idea of a community of mutually supporting ribozymes that multiply together is being confirmed to some extent (Lincoln, T. A. and G. F. Joyce, 2009; Vaidya et al., 2012). Some functional ribozymes have been obtained using RNA polymerase (A. Wochner et al., 2011). Further improvement of ribozyme polymerases led to the production of ribozymes capable of replicating longer RNAs with a specific nucleotide sequence. Improved results are achieved by artificial evolution in ice (J. Attwater et al., 2013). Despite the "division of labor", all models on the cooperative community of RNA, reproducing each other continue to maintain the same problems. As before, the transition 
to the translation system remains incomprehensible. Moreover, the transition is technically not visible in models, it does not have a plausible path and has no theoretical feasibility. In this case, the key reactions necessary for transcription are well reproduced by ribozymes. The reaction of aminoacylation of tRNA is selected in vitro with relative ease, many ribozymes catalyzing this reaction are detected. Some of them carry out aminoacylation with a higher rate and specificity than the corresponding aminoacyl-tRNA synthetases (aaRS) (Turk et., 2010). On the other hand, the construction of phylogenetic trees according to amino acid sequences shows that by the time of the appearance of modern aaRS a large variety of proteins already existed - more than a hundred species. Consequently, ribozymes with aminoacyl-tRNA synthetase activity should have been involved in protein synthesis in antiquity, and the protein aaRS changed them rather late (Yuri I. Wolf, Eugene V. Koonin, 2007). This does not solve the problem, the question remains: how to find a coordinated scenario of early evolution and the starting point for the appearance of the translation system?

\section{An Important Model That Has Gone Un- heeded}

There is only one model capable of solving the paradoxes that have arisen. In addressing the early course of events, this model considers the currently absent ribozyme aminoacyl-tRNA transferases - molecular structures that, unlike protein or ribozyme aaRS, do not use free amino acids, but transfer terminal amino acids from the $\mathrm{N}$ - terminus of peptides to tRNA according to scheme: (AA) $n+$ tRNA $\leftrightarrow$ (AA) n-1 + AA-tRNA. These important molecular structures can be called ribosome-like codons. A group of ribosome-like codos, together with a ribosome, can form a complete translation system, which will differ from the known translation system by the minimum number of protein participants. To form a replication cycle, only one real gene encoding the enzyme replicase (RNA-dependent RNA polymerase) is needed. Replicase is the only true enzyme in the system that is needed to create all copies of the RNA participants in the cycle: ribosomes, ribosome-like groups of codosomes, groups of tRNA and replication genes. The listed molecular components can form a complete self-reproduction system that will function based on amino acid encoding, with the difference that the formation of aminoacyl-tRNA in the system will be accomplished by encoding free amino acids like modern aaRS or their ribozyme analogs, and by coding by transfer amino acids from the N-terminus of the peptides.

A comparative analysis of the protein elements of the modern translation system reveals the need for the existence of an ancient translation system, which should consist essentially of RNA. The analysis shows a long and complex evolutionary pathway of protein translation elements. For the participating proteins to arise, a protein-independent translation system is required, which in its efficiency should be as perfect as the modern one. This means that the listed elements (ribosome and ribosome-like codons) are ideally suited for the role of the ancient translation system. The consideration of this ancient translation system, as the initial cause of the early events, opens entirely new possibilities.

First of all, the reason for the formation of a self-reproducing cycle on the basis of a fully-fledged coding system becomes clear. The study of the ribosome, the analysis of the large ribosomal subunit of $23 \mathrm{~S}$, reveals a hierarchical scenario of its formation. The active center responsible for transpeptidation is an independent domain. It is the $\mathrm{V}$ domain, the oldest part of a molecule that can work independently. This ancient part consists of two blades, which have practically the same secondary and tertiary structure. Presumably, such a structure was formed as a result of duplication of one of the parts (Bokov K., Steinberg S. 2009, Nissen P. et al 2000, Agmon I. et al 2005). It is easy to imagine that if there was an ancient duplication that led to the formation of the most important part of the ribosome, then the same reason could lead to the formation of a whole group of molecules with a similar structure. This is a group of molecules - ribosome-like structures that will have a similar structure to the ribosome and will perform the same reaction. Aminoacyltransfering in the form of transfer of terminal amino acids from the N-terminus of peptides to tRNA (the pathway for the production of aminoacyl-tRNA in the supposed ancient translation system), this reaction is analogous to the peptidyltransferation, which is carried out by the ribosome. These are two similar reactions. The difference lies in the fact that in one case the peptide is transferred to tRNA, and in another, the amino acid to tRNA. Ribozyme codons are a group of related molecules that were formed with a very crude, not exact duplication. Differences in nucleotide sequences, individual parts of molecules, can provide specific aminoacylation, in which each participating codon can encode its amino acid. In addition, coarse duplication caused the formation of a special molecule with large differences, which made it possible to transfer not the amino acid but the peptide; the reaction should be performed in the presence of additional RNA and only if the nucleotides are correctly matched; and finally, this particular molecule was able to release the synthesized protein by hydrolysis - an irreversible reaction that the transpeptidase center of the ribosome carries out independently. As a consequence, a unique irreversible reaction (hydrolysis) between the participants, the group of the codosome on the one hand, and the ribosome on the other hand, will form the specific substrate relationships: the products (aminoacyl-tRNA) obtained in the reversible reaction with the participation of the codosome, serve as a substrate for irreversible synthesis protein on the ribosome. Thus, the key point is that a group of related molecules, due to the regular displacement of the chemical equilibrium of the system, can perform a full-scale matrix synthesis of the protein. Moreover, this action is performed without any additional factors (as is known, there is a nonenzymatic elongation activity of the ribosome) and without the participation of ATP, because the aminoacyl-tRNA obtained in an alternative way is used. Undoubtedly, the efficiency of protein synthesis, in such a system, will be extremely low, only a small part of aminoacyl-tRNA can enter the ribosome. Most of the activated amino acids will return to the structure of random peptides, since the codosomes conduct the reaction reversibly. But if the protein synthesized on the ribosome will not be attacked by the codons due to the internal compact structure, it can accumulate significantly in the system, and of course, among the accumulated molecules, there may be those that will have functional properties. The conservative structure of tRNA for all species and all specificities arose from the evolution of one common ancestor. But the translation system with a single tRNA will not work. However, the primary non-enzymatic duplication that led to the formation of a group of molecules with a ribosome-like structure could be responsible for the formation of groups of similar tRNA-like molecules that would be significantly different due to the primitiveness of the copy. As a result, under the same conditions, under the influence of the same factor, a primitive non-enzymatic process, molecules with similar structure can be formed. These are two types of molecular clone: tRNA-like and ribosome-like molecules, where each molecule has individual differences. These molecules will not have any significance, they are just molecules with a short life span that are formed and destroyed. This will continue until some of the participants in both clones give out just such a combination of molecular composition that forms a cycle of replication. It is paradoxical that for the accidental formation of a translation apparatus, according to this scenario, many different molecules of both clones are needed. Moreover, the more variants of the structure and the more possible combinations that they can create, the loss of a successful option is the more likely. For this it is necessary that both types of molecular "swarm" are for a time together, and their diversity is kept at a constant level. Sooner or later, among the many possible options will be formed the one that will make up the translation apparatus. All this group of molecules will have one important property. It will copy an order of magnitude more accurate than the previous natural duplication. The appear- 
ance of an efficient, efficient transmission apparatus will stop the nonspecific duplication, which will reduce the random generation of the molecular swarm and lead to a gradual replacement of the elements of the "swarm" with the effective elements of the translation apparatus. It can be expected that a group of molecules composing the translational apparatus will copy all its surroundings, its entire molecular swarm of both clones at the beginning, but in the future purifying selection will save from excess. Thus, according to this model, the translation apparatus is formed from a group of related molecules. Its formation has a realistic picture: once the ribosome, with a group of related molecules, meets the RNA sequence of nucleotides in which it makes up information for the replicating enzyme, a cycle of interdependence is immediately formed that can reproduce all its components. As a result, we see a clear rule for the appearance of a self-replicating cycle. Moreover, the formation of protein-synthesizing apparatus based on encoded amino acids has another important advantage in this model. It describes the phenomenon as an integral and one-stage event. And this is the only model that can realistically explain the reason for the formation of a cycle of replication based on protein synthesis in the form of regularity. This moment becomes the key and decisive, it serves as that very starting point for constructing further models reflecting the evolution of the cycle. Given all the above circumstances, it should be concluded that, despite its simplicity and abstraction, this model should be the subject of close attention. It should be viewed from different angles, and its reconstruction, reproduction in an experiment, should become a priority task in the matter of the origin of life.

\section{Molecular Configuration as a Reflection of the Most Ancient Events}

Unfortunately, my competence does not allow me to conduct a correct analysis of the model, to determine the thermodynamic conditions, to give the exact characteristics of the process, to delineate further. But I can not ignore a number of facts consistent with the model. The first thing that attracts attention is the relationship of temperature with the first organisms. The analysis of phylogenetic trees of the three kingdoms of organisms finds deep roots between modern hyperthermophiles (Nisbet E.G. Fowler C.M.R. 1996, Stetter, K.O. 1996). Why do hyperthermophiles lie at the base of the tree of life? There have always been many explanations for this question. The living conditions of the first organisms in hydrothermal sources look attractive. Sources create powerful gradients: temperature, chemical, electrochemical, etc., these powerful gradients can be the driving force of the first metabolic cycles (Wachtershauser G. 1990; Wachtershauser G. 1992; Wachtershauser G. 2006). In addition, hydrothermal sources carry hydrogen, ammonia, metal ions and many sulphides, which are abundantly deposited around. The mineral crust of such sulfites, like graigite, contains microscopic pores. This is a semi-isolated space, a kind of stone cell, into which organic molecules can fall and accumulate. The formed compartments can act as chemical factories for the synthesis of substances (Russell M.J. et al., 1988; Russell M.J., Martin W. 2004; Russell, M.J. 2006). In general, many reasons are associated with the temperature, from the synthesis of the first organic molecules to the formation of functional proteins and polynucleotides. Based on the described model, the shift of the chemical equilibrium in the system should be a regular and repetitive event and it is very tempting to assume that this force is the natural cycles of day and night. Temperature is the driving factor changing the reaction direction (AA) $n+t R N A \leftrightarrow$ (AA) n-1 + AA-tRNA. This reaction is fundamental, the existence of a cycle depends on it. The process can take place in a solid medium with a microscopic accumulation of water that can merge and separate periodically at different periods of the daily cycle. The "day" period is the dehydration of the environment with the formation of small isolated clusters. Completely dehydrated areas are those areas of the environment in which thermal protein syn- thesis and polymerization of nucleotides occur. In the presence of phosphates, amino acids are polymerized at $65^{\circ} \mathrm{C}$, which can correspond to the daytime temperature on the early planet. It is also known that the polymerization of ribonucleotides on a solid substrate of mineral components leads to relatively regular structuring of molecules (Cairns-Smith, 1982; Orgel, 1992; Ferris et al., 1996). The "night" period is the displacement of the chemical equilibrium of the system in the opposite direction, the period of water accumulation and the merging of droplets. All organophosphorus compounds have strong hygroscopic properties, they can accumulate moisture from the atmosphere. Chaotic fusion of droplets extends the cycle, contributing to its entry into other areas of the medium with a rich content of substrate. Such a hypothetical scheme can be used as a model. It is clear from this that the first self-replicating cycle was not a molecularly independent process. The cycle depended entirely on natural, recurring natural phenomena. And only gradually, acquiring and accumulating molecules with useful properties, the cycle could get rid of the natural participation in the synthesis of its components and become a truly molecular independent process.

Consideration of such a hypothetical scheme makes it possible to understand the cause of the structure of the first molecules. So, the first functional proteins, synthesized on the ribosome, should be heat-stable proteins. Thermostability is ensured by a compact structure. This becomes important in the conditions of the functioning of the cycle on the basis of ribozyme codosomes, since the activity of the latter affects the terminal regions of the molecules. As a result, the thermostable structure of the first translated proteins is not only a functional necessity dictated by environmental conditions, but also a way of avoiding the destructive influence of the codosomes.

The ring structure of information molecules is another forced form of structure. The mineral substrate, which is an integral part of the process, introduces a change from the ends of the molecules. It stitches the fragments, ligates, lengthens the end pieces. Under such circumstances, molecules with a ring structure will undergo a smaller change, the ring structure becomes an advantageous form of information storage. The reason for conservative sections of tRNA can be regarded as the reason for the general structure of those structures that work with them. In general, any theory the emergence of life, one way or another, should explain the nature of the structure of the first molecules. Of course, the model is absolutely speculative, it is not supported by model calculations, and, nevertheless, there is every reason to believe that this model explains the nature of the structure of the earliest molecules, it explains their better preservation under the conditions of the initial functioning.

In addition, there is another very interesting circumstance that can cause structural similarity of the participants in the cycle. This is the stereospecificity of amino acids. All participants in the cycle have a common structure of the catalytic center. Each ribozyme codosome may be able to transfer only a certain optical isomer of the amino acid from the $\mathrm{N}$-terminus of the peptide to tRNA, even if the primary protein-peptide mixture consists of an equal number of different isomers. Thus, the reason for the formation of amino acid steriospecificity can be the result of the general structure of the ribosome and the codosome. It looks quite realistic, because to recognize another optical isomer it is required to have a fundamentally different structure of the active center.

\section{The Concept of an Infinite Replicator. The Principle of Preserving the Relationship. Cell Selection.}

The above-described model of the formation of a translational apparatus consists of extrapolation and assumptions, the model in great need of detailed elaboration and careful analysis. Now this is only a hypothetical scenario. But this scenario is very attractive, it 
has a concrete and understandable mechanism leading to the formation of the translation apparatus. This is the primary replicator; its formation becomes an inevitable and natural event.

When we study the issue of the origin of life, we neglect the possibility of the existence of a regular course of events, we perceive the course of events solely as an accident. Development is understood as such a course of events, which is based on competitive relationships or some chemical advantages, stereochemical relationships, etc. Attention focuses on the question "how does this happen?" The focus of attention is directed to the study of small molecular circumstances. In this case, an entirely different situation develops when development is viewed from a full-fledged translation apparatus. The apparatus can vary in molecular composition, its components can also vary, but the device always retains its structural functionality. Stable preservation of the constructional functional is not an assumption, it is a fact containing certainty, the basis of this confidence is the fact that life has never been interrupted. The central question in this case is "why can this happen?", "How is the long-term preservation of the translation apparatus possible?" The answers to these questions should not be sought in small details and molecular circumstances. The approach requires the consideration of events exclusively in models of the behavior of molecules. These models should demonstrate for what reasons the once-generated translation apparatus can persist continuously. This is no longer a question of small details, it is a common question in which the details and circumstances can be arbitrary. In this case, it is possible to verify the truth of the model only for the key points that should be highlighted when studying the model. If the characteristic elements of the model correspond to our observation from life, then such a model can be considered true. I call this the starting point when studying the origin of life. The position is simple, and it is very important to understand it. In the classical approach, the solution of the problem is seen in separate molecular details, preserving the replicator is perceived as a problem that must be solved. In another approach, preserving the replicator is considered not as a problem, but as a fact, and the reasons for preserving the replicator become the object of research. Attention is focused on the conditions under which the long-term preservation of the functional of the device becomes possible. This is a fundamental difference in approaches These are two different views on the same topic. In one case, events are interpreted within the Darwinian understanding of the course of evolution, and in another case, events are viewed in a broad, abstract sense. Scientific principles allow to approach the solution of the problem from different sides. There is no contradiction or deception in this, it is the same full-fledged scientific approach as in the first case. The approach is revealed in the ideological concept of the "infinite replicator". Once formed functional connection between proteins and nucleotides is in such conditions, under which it is always maintained throughout the entire precellular period. It's about the connection, not the molecules that make it up. The connection has never been interrupted in absolute terms. It still persisted and was not violated in absolute terms ever in spite of whatever molecules this connection was, and no matter how small a number of molecules this connection was not determined. The most important principle in this topic is the principle of preserving the relationship. The elucidation of the material basis for the realization of this principle is the main task in the matter of the origin of life. At the present moment, in the cellular world, the principle of preserving interconnection is realized at the expense of its material basis, it is composed of gene flows with predominantly vertical distribution. Does this mean that in the early period, there was the same material basis? Does this mean that the principle of preserving the relationship was implemented in a similar way, through vertical gene flows? If we build on this belief, this will undoubtedly be a mistake for us. We need to consider and propose other reasons that will not be related to vertical gene flows, and at the same time, will be able to explain the reason for preserving the functional apparatus of translation for a long time.
Let's pay attention to the "chasm" that separates the replicator from a full-fledged cell. How can a replicator overcome it? Can we describe this path by any known evolutionary regime? If the replicator turns into a cell due to other processes that are unrelated to the biological world, can we consider these events as a molecular evolution? Obviously - yes, we can call this path a molecular evolution, since the whole issue of the origin of life is a matter of finding traces and evidence of the replicator becoming a cell, regardless of the structure and forces that lead to it. The search for answers is not limited to the biological perception of the problem, we have on hand a classic puzzle game with a simple and absolutely realistic solution.

The following theoretical questions help to understand the reason for the replicator's transformation into a cell. Can the resulting link between proteins and nucleotides continue indefinitely or endlessly? What do you need to purchase or develop a replicator for connection to reach infinity level? In this question, the essence is clearly seen, the motives for achieving the goal become clear. A cell is an independent, isolated replicator that uses limitless resources of low molecular weight substances, uses inexhaustible sources of light energy or chemical reactions. A cell is, first of all, a replicator, which has almost reached the level of infinity. This approach to the problem brings the whole issue of the origin of life into the field of theoretical constructions. How can a replicator be able to reach the level of infinity, using all the imaginable variety of translated molecules to achieve the goal?

Answers gives a very simple model of the behavior of molecules. The sequence of events can be as follows: the replicator populates cells with an individual set of RNAs and is isolated for some time. The mineral substrate, which was mentioned earlier and was responsible for the correct structuring of the oligonucleotides, provides a variety of translocated molecules for each cell. Penetrating a cell that has an individual set of RNAs, the replicator is tested for the stability of the relay apparatus with new contents. The replicator is tested for the compatibility of its elements with individual RNA cells in each cell. If the combination with new molecular elements fails for the replicator, if such a combination makes it difficult or significantly reduces replication, such a molecular variant will not be further developed. It will be destroyed. Life is a constant construction of molecules, the speed of formation always exceeds the rate of their destruction. All this is true for the early period of development. As soon as replication stops in the cell, RNA will naturally be destroyed by hydrolysis, and such an untenable replicator will be molecularly destroyed. It can be expected that most of the "cell" combinations will be lethal for the replicator. But if in any cell the replicator still persists, if a portion of the new RNA cells allow the replicator to create multiple copies, then such a molecular variant of the replicator will continue its way. The replicator with its numerous copies will populate adjacent cells, and in each of them will be tested again for the combination of their components with new portions of the individual RNA cells. This is a general scheme of molecular selection, which can function at the expense of natural cycles like "day and night." To some extent, it is appropriate to draw an analogy with the method of selecting aptomers with given properties. In both cases, the power of selection is the driving force. But if SELEX is aimed at molecules with given properties, then a group of molecules is subjected to selection, which is selected according to the principle: whether replication is preserved or not. The probability of a successful combination of a replicator with new molecular components will obviously be low, however, if the principle of maintaining the relationship exists, it will not allow the system to be reset. Whichever small number of replicators is left, it will still spread and will be ready to be tested again, constantly passing a lot of "bottlenecks". Such a sequential course of events in the form of cell colonization and testing leads to the gradual accumulation of potential genes from the entire area of the environment. The environment forms a genomic library with all its many cells. The genomic library is the primary reservoir of genes. When the cycle is changed, the library is updated every time, cells with an individual 
set of RNAs become an endless source of options for new genes. If the process is repeated several times, the same cells are reused, then, after several cycles, the replicator starts to meet the "glued" molecules, which were once elements of the old structure, but were unsuccessful and hydrolyzed, and then chaotically ligated to mineral substrate. Now these are new elements of the library, which were formed from old insolvent variants.

Let's pay attention to what stands behind such a simple course of events. First, evolutionary innovations can be formed not only as a result of gradual, small changes, but also in a spasmodic (cell) way. Not only all known elementary evolutionary events of genes, such as replacement of nucleotides, insertions, deletions, duplications, etc., can be realized, but also a very important form of selection of an entire group of molecules in a single cell. This selection is unique, it does not occur in biological systems. It can only go based on an active, workable, translational apparatus, to which new versions of the translated molecules are simply added. This circumstance allows the replicator to obtain evolutionary innovations while retaining the very mechanism of expression at a sufficiently high level of accuracy. In fact, a very simple technical solution in the form of the addition of potential genes to the operating translation apparatus in a variety of separate isolated attempts makes it possible to bypass the problem of the concept of the error threshold, which is undoubtedly the most important advantage of the "cell model".

Secondly, it is possible to clearly observe which selection dominates. Molecular selection is not only the consolidation of favorable changes. The key role in the events is played by purifying selection, in this case it acts through the hydrolysis of RNA, fragmenting the untenable variants of replicators. RNA is best suited for this role as a less stable molecule. The screening of variants that are incapable of efficient replication by hydrolysis constitutes the physical essence of natural molecular selection. This is a simple and objective reason for the organization of molecules. It can be assumed that the process of molecular organization is composed of many random events that he has not initially any given value, but due to a set of isolated attempts, resulting in a lot of trial and error, the selection will inevitably lead to the accumulation of molecules with functional value that becomes the reason for the organization of molecular composition

Thirdly, in case of "cell selection" due to repeated use of cells, a mechanism is formed that leads to the block structure of potential genes. It is believed that the multi-domain architecture of the protein is the most important reason for the emergence of novelty in evolution (Levitt, M. 2009). Spontaneous reactions of rearrangement of nucleotide sequences that occur without the participation of any enzymes or ribozymes are known (Chetverina et al., 1999). The exchange of nucleotide sites can pass between different molecules or within one. These reactions are spontaneous and nonspecific in respect of nucleotides and nucleotide sequences and can occur anywhere in the chain. However, the speed of such rearrangements is extremely small - one event per hour per billion nucleotides. Undoubtedly, spontaneous rearrangements make a definite contribution when shuffling fragments, but this contribution will be incommensurably less than one can expect from a combination of hydrolysis and ligation of fragments on a mineral substrate. Cutting molecules and their further bonding is really a very effective way of rearranging molecules and this mechanism is seen as the main mechanism in the formation of new variants of genes. Reorganization takes place in each cell, it precedes the replicator population and is carried out from old, often useful parts, which significantly contributes to the speed of evolutionary innovations in the form of neofunctionalization of old workable units. At the same time, hydrolysis and mineral substrate bear a multivalued significance, they complement each other and logically fit into the general architecture of events.

Fourth, one of the characteristic features of the early period is uncontrolled horizontal gene transfer (Woese, C. R. 2002). The cell scenario corresponds well to this fact, the fusion of microscopic accumulations of water is propagated by the replicator. If several replicators are stored at once and each of them passes through its "bottlenecks", then a network will be formed between them for mutually beneficial exchange of innovations.

\section{Molecular Evolution Close-Up.}

If we continue to consider the events in general terms, then it is not difficult to understand that the path that the replicator performs in order to turn into a cell is not the only one. The replicator can overcome the "chasm" in different ways. It can use different genes and function proteins to achieve the goal. Different ways of carbon utilization, different ways of DNA synthesis, different ways of lipid synthesis, etc. All this clearly demonstrates how replicators can come to the solution of the same fundamental problem by ways that are independent from each other. The fulfillment of key biological functions is carried out by a huge variety of unrelated enzymes (Omelchenko et al., 2010). The results of comparative genomics show that the replacement of nonorthological genes and the loss of genes are so widespread that only a small number of functions are monomorphic and ubiquitous. The universal kernel of life, which can be found in any organism, disappears a little. All that is truly universal is about thirty genes of translation proteins, three large subunits of RNA polymerase and, approximately, the same number of structural RNAs in the form of rRNA and tRNA (Koonin, E.V. 2003). The nucleus of cell life, which can be found in almost all genomes, consists of about 60 genes. The smallest set of genes that will be sufficient to support the simplest cell, under the most favorable conditions, consists of approximately 256 genes, which is only 6 times less than the minimum set of genes necessary for the existence of an autotrophic organism. Taking into account the possibilities of the described model, it looks quite realistic that in many isolated attempts, moving from one cell to another, the replicator collects the missing genes, "scoops" them out of the boundless primary medium reservoir and does it repeatedly in different populations.

If we continue to look at the course of events in general, a very interesting picture emerges, according to which the molecular acquisitions for the replicator are absolutely random, but the selection factor forms an orientation, it forces "to collect" those genes that are useful. When observing the principle of preserving the relationship (that is, preserving at least one cell with an effective replicator), selection in cell conditions inevitably leads the replicator to a more perfect version of the structure. If a portion of the new genes in the cells brings some advantage to the replicator, but as a result, a little more copies of the replicator will be formed, then due to the features of the system functioning, this advantage will become the dominant variant of the replicator structure. In such a system, any useful achievements are picked up and distributed. As a result, all beneficial changes, such as expanding the substrate field, increasing the speed and efficiency of replicating copies, are maintained and fixed in a natural way. In contrast, unprofitable innovations that disrupt or reduce reproduction of the replicator are destroyed by hydrolysis, i.e. there is a physical destruction of those genes that bear untenable options for reproduction. Later, their chaotically glued parts become new variants and again tested in the following attempts. It can be expected that different in molecular composition replicators will accumulate different useful abilities - genes that code functional proteins and at the same time all this community simultaneously coexists, randomly populating different cells of the primary medium. It is quite right in this case to consider and reasonably approach the issue as to a single universal ancestral state (LUCAS), a set of various replicators with a non-constant, except for the replication nucleus, the gene composition. The fusion of replicators unites genetic material, which can lead to the accumulation of disparate elements, forming a single, integral, functional system and as a result of their unification - the "crystallization" of the basic features of the cell expressed by C.Woese. 
In general, when considering the theoretical model of the organization of molecules in cell conditions, it is precisely the pattern of events that has already been repeatedly expressed, namely, the notion of a non-cellular inconstant compartmentalized community of various replicators of inorganic cells that live in networks (Martin W. Russell 2003, Koonin, EV, Martin W. 2005). There is also a variant with terrestrial networks of geothermal sources, the socalled "zinc world", which explains well the reason for the high content of zinc in ancient proteins and enzymes (Mulkidjanian, A. Y. 2009). Moreover, as stated earlier, this community is a community of virus-like agents, which at an early stage did not yet have DNA and lipids (Koonin E.V., 2009). In fact, as the host cell provides everything necessary for the reproduction of viruses, so natural conditions take on the missing functions of the replicating cycle and provide it with everything necessary, creating optimal conditions for reproduction of copies and distribution. In fact, the gene repertoire at an early stage under such conditions will be limited solely to components for gene expression, which fully corresponds to the reason for missing components in the reconstruction of LUCAS.

If we continue to consider the situation in general terms, we can understand why selection leads to the appearance of such important components as DNA, lipids and nucleotide triphosphates (NTP). In the classical approach, it is commonly believed that the reason lies in a competitive relationship. In fact, there are other reasons related to the peculiarity of the system. The whole molecular evolution is subject to a single rule - the replication core, which is functionally dependent on the cyclically changing environmental conditions, is the driving force of the process. Spreading through the cells, the replicator breaks down where the unsuccessful molecular combination develops and creates its numerous copies where the combination allows it to do so, then it spreads and the cycle repeats. The principle is simple: if the replication core stops, then all generation within this combination line stops, but if the generation continues, it leads to the optimal result. The course of events includes an incredibly rapid drift of genes. In fact, only one nucleus of replication remains unchanged, all RNA molecules not included in the replication core constitute a rapidly changing composition. This circumstance does not allow us to stop selection on the achievement of a dense, compact, ideal replicating machine in all respects. Uncontrolled drift of genes is the most important feature of the cell system. The high rate of molecular new acquisitions, against a background of rigid purification selection, sifts many variants of the replicator structures for their rare useful properties, which very quickly become a property for all and already in different populations are again sifted in a variety of separate isolated attempts. The central point is that this situation will continue until the replicator, by its structure and functionality, changes the conditions of selection dictating to it. The replicator can change the situation only if he finds an opportunity to develop such constituent elements that will enable him to obtain independence of the production of his copies from the external environment, to form isolated isolation from the outside world, and to translate his information into a more reliable form of storage. Paradoxically, the fact that whatever structure has a replicator, being in cell conditions and subject to cell selection, it constantly changes its composition (with the exception of the nucleus of replication) throughout the period until it turns into a cell.

It is possible to designate the entire "situation with cells" as a single mechanism by which the replicator is forced to increase its complexity. The complexity of the structure and organization of the replicator will grow due to the constant testing of the material. It is important that the increase in complexity will continue until the level of organization of the replicator surpasses the limiting factors of functional conditions, which can occur only when the replicator finds a way to synthesize DNA, lipids and NTP. Thus, throughout the entire pre-cellular period, the replicator is forced to increase the test material, the build-up continues until such an organization of the replicator component composition that will be characteristic of the cell is formed.
We can distinctly observe that the whole molecular evolution is a separate, independent, natural phenomenon. This phenomenon has a structure and boundaries. It must start with the replicator and end with the formation of the cell. Awareness of the fact that cell formation is a separate, independent, natural phenomenon opens the door for the study of the issue in two ways: either by mathematical modeling of the organization of molecules, or by reconstructing the course of events at the molecular level, where the sequence of events will be nothing more than a reflection mathematical model.

\section{Mosaic Picture of Molecular Events.}

Let's pay attention to the border of the phenomenon, the exit from the environment is the moment when the replicator reaches a special level of organization, in which he gets the opportunity to get rid of external dependence. In the classical approach to the problem, this event is not really given any importance. The event is perceived as an "outcome", as a certain change of residence, such an underestimation of events does not allow us to understand the depth of the question. Since the release from the environment, with the termination of cell selection, the biological world will begin. Up to this point, all events, the whole set of events, are a single, integral act of ordering microscopic events into a macroscopic level. The organization of molecules is a separate natural phenomenon, which has its own rules and is subordinated to its principles. A dense sequence of random events makes the process move to the formation of a cell. In this regard, the question: "what would we see if we lost the record anew?" It can be said with absolute certainty that the result will be unchanged. Expectations will be justified, a full-fledged cell will be formed, although it will include other molecular components. Before the replicator, on the way to its transformation into a cell, there are quite specific goals: the transition to irreversible protein synthesis, which becomes possible with the use of free amino acids; concentration of genetic material in one compact, reliable and stable form of information storage; application in the synthetic processes of a universal energy source; formation of functional membranes and insulation. It's a long way, it consists of many steps. Each step along this path is a separate, useful, molecular acquisition that gives the replicator an advantage and which very quickly replaces other variants of the replicator structure. Perhaps only a pre-cellular biota from all evolutionary biology can carry the opposite answer to Gould's question. In this case, the expediency of producing the necessary elements is dictated by the very necessity of having such elements for further existence.

Let us pay attention to the features of the system leading to the ordering of the molecular composition of the replicator. If these were the usual selection conditions and the selection was determined solely by the acquisition or loss of molecules, then we would observe in this case the degradation of the replicator. In contrast, selection leads to a more complex organization of the composition. The cause is external influence. It can be observed on the example of microorganisms as external factors contribute to the development of previously not inherent abilities. Long-term follow-up of E. coli shows how new possibilities are being developed and new abilities are being discovered, for example, in the form of citrate utilization (Barrick, J. et al., 2009). According to the authors of the project, long-term experimental observations can be considered as a model for the development of new complex abilities during evolution. At the first stage of the "potentiating", genetic changes that do not play a special role are accumulated, but later in the "actualization stage" these abilities are realized and, if they are useful, they are improved. Unlike modern organisms, early replicators had much more opportunities to identify new abilities. Their genetic repertoire, except for the replication core, was not permanent. Replicators were forced to respond to changes in the composition of the environment. The original composition of the replicators was probably very meager, its molecular components were limited exclusively to the most necessary elements for 
reproduction. At the earliest stage, there could not exist a whole series of amino acids with important functional groups, there were no coenzymes and many metals. The transcribed proteins had a limited range of catalytic reactions and, in most cases, represented "apoenzyme debris". This is a companion material that should not reduce reproduction. Catalytic capabilities at an early stage were greatly limited by the primary composition, for the effective replication of the replicator sufficient reactions of a transferase nature. A scanty molecular composition is advantageous at an early stage, because it increases the likelihood of implementing the principle of preserving the rconnection.

All this is consistent with other models. There is a model of early genes with a rich content of $\mathrm{GC}$ nucleotides, which gives a triplet periodicity in the form of SNS sequences (where S is G or C, and $\mathrm{N}$ is any nucleotide). According to this model, the first amino acids must be encoded by codons with the general formula SNS This requirement is met by 10 amino acids (Gly, Ala, Val, Glu, Gln, Asp, His, Pro, Arg, Leu). The list can be narrowed to 4 (Gly, Ala, Asp, Val) and presented as GNC codons. The model for the emergence of the genetic code through the stage of the GNC codons was called the GADV theory, according to the first letters of amino acids in a single-letter expression (Ikehara K. 2002, Ikehara $\mathrm{K}$ et al. 2002). Four amino acids provide 4 of 6 conditions for the formation of sufficiently diverse structural and catalytic properties of proteins-hydrophobicity, ability to form $\alpha$-helices, $\beta$-folds and bends (Oba T. et al. 2005). In experiments on abiogenic synthesis, it is these amino acids that are formed in the greatest quantities. (Miller, S.L. 1986). Moreover, a high content of GC-nucleotides provides higher accuracy of RNA reproduction. The high content of GC in genes reduces the probability of encountering a stop codon, thereby facilitating the formation of sufficiently long polypeptides capable of exhibiting functional properties. One of the characteristics of hyperthermophilic proteins is high charge density, which in the SNS code is represented by both negatively charged amino acids (Asp; Glu) and positive (Arg, His). In addition, it is known that if the genetic year is "doublet" and the third position does not matter, then such code is more resistant to errors, it can be even more reliable than modern (Novozhilov and Koonin, 2009). It can be assumed that in the early period there were several ways of forming genes. Duplication followed by neofunctionalization is the way that exists until now. But in the early period, there could be at least two other possibilities. This involves the involvement of genes from the primary reservoir of the medium and an increase in the information capacity of already existing genes by increasing the significance of nucleotides in the triplet. In the latter case, any changes will affect all participants in the replication cycle, and it can be carried out, and in the case of a positive effect, be preserved, but only when it satisfies all participants in the cycle at once, i.e. pass synchronously, affecting the entire molecular ensemble of the replication core. Studies of the genetic code show that the code has evolved to minimize the consequences of errors. When selecting from the possible codes, only one out of 100 alternative codes has no less noise immunity than the existing one. If additional restrictions are introduced that take into account actual trends in the nature of mutation and the occurrence of errors in the translation, then only one in a million is better than the canonical one (Freeland and Herst, 1998). In addition, mathematical models demonstrate the universality of the genetic code as a result of copious horizontal gene transfer (Vetsigian et al., 2006).

Most enzymes for catalytic activity require coenzymes and meta ions. These important constituent elements could enter the system at different time intervals. External factor, penetrating into the cells, cause the discovery of the act of catalysis in some proteins among "apoenzymatic debris". At the same time, whatever reaction is disclosed, it has the ability to survive, but only if it does not violate replicator reproduction. Otherwise, a response that does not contribute to the preservation of replication will lead to the destruction of this option. Stopping the playback will mean the destruction of RNA and the same version of the gene that was responsible for the given garbage protein. The arrival of an external factor corresponds to the "pandemic" scenario, which spreads through the cells and affects each replicator. A replicator that will be able to benefit from this, preserves, spreads and populates other cells. The useful gene and the revealed reaction become an integral part of the replicator. They increase its effectiveness. In fact, the entire development of the replicator depends on the variety of "garbage", which is individual for each cell, and on the number of independent, isolated attempts, i.e. number of cells populated with replicators. If these conditions are achievable, then as a result of simple cutoff selection, we will be able to observe how the replicator gradually accumulates precisely those molecules that will complicate it and increase the efficiency of reproduction. If the input of the external factor passes in stages, the "epidemic" replaces abundant reproduction, followed by another wave of destruction, then the result of this whole series of events will be something other than the accumulation of useful genes. The number of useful genes will increase with each wave, as each surviving replicator spreads with all the genes useful to it. If this happens repeatedly, the result of this whole series of events will be the growth of functional genes. Thus, genetic selection is associated with the disclosure of catalytic activity of the enzyme and this occurs under the influence of an external factor. The selection separates the gene from the "garbage" and separates exactly the gene that becomes useful to the replicator. Moreover, this is the simplest mechanism of concentration of functional genes. This circumstance corresponds well to reality, so genetic studies show that there are wide intergenic sites in the genomes of eukaryotes, and there is a huge density of genes in earlier genomes, prokaryotes and viruses (Koonin, 2009).

Thus, a very simple conceptual ploy in the form of an "infinite replicator" makes it possible to understand the course of historical events. While this picture has only a mosaic, fragmentary character, this is just a description of the model, a kind of plot of the picture. The content of the picture is all that carries a semantic load in the form of molecular innovations based on randomness. The main events unfold in the three main lines leading to DNA, to lipids and to the use of free amino acids in matrix synthesis. The chains of events are interrelated and closely intertwined. It is important to perceive them as products of natural molecular selection that leads to them, and when they reach it stops.

\section{Evolutionary Steps and Chain of Events}

Probably, the first steps on the way of the replicator to the cell are performed by its viral nature. Synthesis of a simple spherical capsid, which can be formed by self-assembly of a single protein, better ensure the safety of RNA - a step that carries a certain advantage. This step is also the first significant division into population groups: viruses and virus-like replicators, the main treasure trove of further development.

Let's look at the first chain of random events. It goes from RNA organisms to DNA-organisms. The size of the RNA genome can not exceed more than 35 tons of nucleotide pairs. If this situation continued, it would severely limit the possibilities for development. RNA is easily hydrolyzed, cytosine is gradually deaminated, turning into another base, uracil. In addition, RNA, when forming hairpins, forms catalytically active sites that catalyze self-cutting. It is possible that the early replicator was helped by the redundancy of the genes during the fusion. It is also possible that everything started not with RNA, but with methyl RNA, which is more stable (Poole A., et al., 2000). DNA has no such deficiencies, and therefore its advantages are undeniable. Synthesis of deoxyribose is carried out by at least three families of reductases, they are similar in sequence, but differ in the reaction mechanism and in coenzymes. Oxidation-reduction reactions require copper, iron or ironsulfur clusters. Global genomic analysis shows that in a short period of time there was a surge in gene production, called the Archaean expansion. In this period, oxidation-reduction enzymes, 
ferrous-containing enzymes are formed (David, L.A., Eric J. Alm 2011). Enzymes, functionally associated with nucleotides, have a more ancient origin, they existed before the expansion. The receipt of new components in the system, as already noted, provokes selection according to the scenario of the "epidemic". As a result of the selection, useful enzymes are preserved, become an integral part and in the future can become a property for all population groups. The accidental formation of enzymes such as ribonucleotide reductase and thymidylate synthetase (the latter also doubled independently) opens up a direct road to DNA. For a replicator, DNA synthesis has an obvious benefit in the form of an increase in the size of the genome and complexity of the organization. However, to realize this benefit, other molecular accidents are needed, which are surprisingly repeated in history at least twice. A comparison of bacterial and archaeal enzymes reveals the least similarity in the most important components of replication-DNAdependent-DNA polymerase and primase (Leipe et al., 1999). The DNA polymerase of archean and eukaryotes is clearly derived from viral reverse transcriptases and probably have even more distant relatives in the form of RNA-dependent RNA polymerase. Bacterial DNA polymerase has no close relatives, and among distant ones it has a connection with nucleotide transferases (Koonin, 2006).

The second branch of random events is more complex, and its links are not so obvious. This chain of events leads to the use of free amino acids in the matrix synthesis of the protein. The process requires ATP. It is not very clear that in fact served as a source of ATP. It is possible that ATP was formed in some substrate phosphorylation reaction, or ATP was formed by transfer of phosphate residues from polyphosphates at high energy level, as modern kinases do, transferring phosphate residues between nucleotides, and polyphosphates could be formed under the same conditions. It is obvious that NTF began to form very early. Excess energy in the synthesis of the main components gives an advantage, this makes the reaction irreversible, independent of environmental conditions. The replicator arose in cyclic processes and could exist due to a regular cycle change at least until the moment when a more efficient way of forming copies was formed. The use of macroergic phosphate becomes a very important achievement for the replicator. After that, the replicator can create its copies continuously, regardless of the phase of the cycles. It seems that the common ancestor discovered this ability very early and started using NTF very early. Nucleoid-binding domains of GTPase and related ATPases have an extensive family. They all contain a characteristic phosphate-binding domain (P-loop), a conserved motif that was fixed at the earliest stages of evolution (Gorbalenya and Koonin, 1989; Trifonov et al., 2006). This stacking is also the most common domain in all prokaryotes (Wolf et al., 1999). It is important that the evolutionary analysis of this stacking suggests that it preceded the modern translation system. All aaRS are divided into two classes with unrelated domains, class I has the Rossman stack, class II biotin-synthase. Both domains are widely diversified by different proteins. Comparative analysis leads to the conclusion that a huge structural and functional variety of proteins (more than a hundred species) arose, even before the modern translation system appeared (Wolf, Y.I., Koonin, E.V.2007). Of course, all these facts testify to the existence of the original ancient translation apparatus, which was accurate enough and effective, and already on the basis of this apparatus the translation system that we know is formed.

All existing aaRS are very different. They could be formed gradually and even in isolation in different populations. aaRS could replace ribosome-like codons by a known evolutionary replacement scenario for nonorthological genes - when a gene with a duplicating function appears, the old gene is released and can be lost. Inclusion of free amino acids in the coding system could be carried out over a long period. In many eukaryotic bacteria and organelles there are no specific aaRS for Asn (asparagine) and Gln (glutamine), in a number of archeas there is no specific aaRS for Cys (cysteine). In these cases, the synthesis takes place with the participation of nondiscriminant synthetases that are able to attach their amino acid to foreign tRNA, and then they are amidated to form Asn-tRNA and Gln-tRNA. Synthesis of Cys-tRNA can also take place without the participation of the corresponding aaRS. The pathway consists of erroneous aminoacylation of tRNAcys with phosphoserine (Sep) phosphoseril-tRNA-aaRS followed by a tRNA-dependent conversion of Sep to Cys (Yuan J., et al., 2006). Non-standard amino acids have their inclusion pathways and their tRNAs, so selenocysteine (Sec), has at least two different ways of sec-tRNA formation. Pyrrolizine (Pyl) - has its own tRNAPyl and its pyrrolizyl-tRNA synthetase (Pyl-APC-azu). The presence of such "non-standard" aaRS as Sep-aaRS, Pyl-aaRS, and two types of Lys-aaRS (I and II types), and no less importantly, the possibility of obtaining aminoacyl-tRNA in various reactions suggests that the inclusion of new components in the coding system is not so rare and unique. Most of the existing aaRS in the formation of aminoacyladenylate do not require tRNA, only some aaRS are truly tRNA-dependent. It is possible that all aaRS were once functional, that all of them were once capable of carrying out the reaction, but they did not make up the coding system. In this form they could be part of the accompanying material, the replicator reproduced them, but they were not part of the replication core. Accompanying the replicator for a long time, they could separate, small, attempts "wedged" into its core. If there are many such attempts, then gradually, the coding system will change, an optimal variant will be formed, and the principle of interconnection will not be violated.

For changes in the genetic code, new variants of the structure of tRNA are needed. The replicator was formed from two types of molecular "swarm". Even before tRNAs turned into independent replicative units, this swarm could include thousands of such molecules, and the translation apparatus itself could contain hundreds of variants of isoacceptor tRNAs. Then the first steps of the evolution of the genetic code were carried out due to the diversity of tRNA, their "mutational background" provided a choice when optimizing the code. Frequently encountered elements of tRNA or their parts could act as signal marks. Thus, in the replication of RNA viruses and retroviruses, tRNA-like structures are involved. Some viruses have similar structures at the ends of the genome. Other viruses use tRNA as a primer for the synthesis of the first chain. The activity of the codosome and ribosome is related to the CCA fragment, which could often be found among tRNA-like molecules. In the maturation of tRNA, one of the oldest ribozymes, RNase P, takes part, it cuts off the ends of immature tRNAs and removes, among other things, the CCA fragment. The CCA fragment is then rearranged with a separate enzyme, the nucleotide transferase. The sequential course of events, in the form of removing labels from all molecules and establishing it only on selected molecules, corresponds to an act of bringing order with a molecular swarm. This is an important step that gives a significant advantage. In history, this happens twice, the nucleotide-transferase was at least twice formed. In bacteria and archaea, these enzymes have different origins (Stefan Vortler, Mario Morl. 2010). In addition, there is a close relationship of these enzymes with other telomeres adding only adenine. In this case, some viral and even cellular polymerases add extra adenine to the end of the chain. Genomic tags can be regarded as archaic evidence of the once existing molecular swarm.

Moreover, it is possible to observe other traces of the primary molecular "swarm" in ribosome-like structures, which at the earliest period should also have a lot. Not all molecules could have functional significance, but the replicator was forced to reproduce them all. They all accompanied the nucleus of the replicator, as a result of which they often served as sources for new variations in genetic material. Different nucleotide sequences characteristic of these structures could be included in the most diverse genes of the replicator. This circumstance reflects the ribosome. Thus, in the rRNA sequence, using certain sophisticated methods of analysis, certain rudiments are revealed: parts of the structure of tRNA, traces of coding of ribosomal proteins, traces of enzymes encoding 
protein synthesis and energy supply (Root-Bernstein M., RootBernstein R., 2015) "dissolved", and its small fragments became part of many genes, as evidenced by the ribosome.

The third chain of random events leads to membranes and the associated energy. The formation of membranes creates a lot of problems, especially the problem of "hunger". This difficulty can be overcome by a long preparatory stage. It is similar to the "potentiating" stage, which is observed in long experiments for E.Colli. Obviously, only some populations of replicators can get on the right path and achieve a successful membrane formation. The correct way will be one in which the replicator will synthesize proteins before the synthesis of lipids, which will help to get rid of the problems associated with the membranes. All other populations of replicators that do not go through the "potentiating" stage are doomed to extinction. In the reconstructed genetic set of LUCAS, it is the common membrane proteins such as membrane ATPase, the secretion system of type III proteins, the signal recognition particle subunit (SRP), whereas the membranes of bacteria and archaea themselves are different, they have different chemical compositions and different synthesis routes (Pereto et al 2004).

The idea of a single ancestral membrane is experiencing great difficulties. Different isomers of glycerin create a physically insoluble membrane, and only single-tail lipids from terpene alcohol and a polar head without glycerol can be formed from common enzymes that bacteria and archaea have in lipid synthesis (Dibrova et al 2011). These membranes are "leaking" for ions. The properties of membranes strongly depend on the inclusion in their composition of various additives, the length of the hydrocarbon chain, the number of double bonds, the chemical modification of lipids in the form of cross-linking the ends of hydrophobic tails of lipids from the inner and outer sides of the membrane, etc. Different organisms, being in different habitats, actively use all this wide arsenal. It seems very strange that early organisms did not choose a change in the properties of the existing membrane, which would be a logical and expected option when adapting to new conditions. Also extremely dangerous is the fact that early organisms having a common membrane are twice solved for their complete replacement. Moreover, it is technically difficult to imagine how a replacement could be performed at all, while preserving the functions assigned to it. If we adhere to the previously stated principles according to which only a rigid purifying selection can bring the replicator to the goal, then it can be considered that the conditions must be changed dramatically and only then the accidental molecular accident can be favorable to conservation. In this case, both the membrane structure and the structure of the device in the form of an ion pump will be preserved simultaneously.

The main subunits of ATPase and their mutual arrangement resemble the system of secretion of type III proteins, this is common for bacteria and the archaea system actively secrete proteins from the cell. The other part of the device is a stator, it has a precursor in the form of a helicase, an ancient family of rotating ATPases. Both families F (bacterial) and A / V (archeal and vacuolar) ATP synthase are homologous, but the rotor rods necessary for the rotation transfer are of different origin, indicating that both families of ATPases were formed twice, two different proteins were stuck in system of secretion and both times the apparatus of effective transmission of rotation was formed (Mulkidjanian AY et al., 2007). A sharp change in the ionic composition is consistent with the primacy of sodium energy. Sodium ATPase occupies the lowest branches on the phylogenetic tree. Proton ATPase most likely appeared later as a result of changes in the sodium (Mulkidjania A. Y. et al., 2008; Mulkidjanian AY, et al., 2008). As mentioned earlier, the process of organizing molecules will be stopped at a time when, the level of organization of the system will exceed the limiting conditions of the process. Energy-dependent control over ion concentration in an isolated discrete unit leads to conservation of the system. This circumstance becomes a necessary functional requirement, since the whole system of enzyme selection was carried out due to changes in the composition of the medium. Now this composition will be virtually permanent. We observe the same ionic composition in bacteria and archaea, although they diverged almost 4 billion years ago. The concentration of such ions as potassium, sodium, zinc, phosphate is unchanged and constant. It has not changed throughout the history of life, and their ratios are even more conservative than any other molecular components (Mulkidjanian A.Y., et all 2012).

\section{Conclusion}

It can be concluded that all science makes a circle. She goes a long way to go on to an understanding of the course of events that can be obtained by investigating the issue directly. The model, which is least of all paid attention, on closer examination, most corresponds to the existing reality. My modest explanation is intended to show how facts are linked into a conceptual thread. This thread begins with the formation of the translational apparatus and ends with the formation of the cell. The whole course of molecular events is seen in the model of the "infinite replicator". At first glance, this model looks delusional and absurd ideas, but it contributes to the formulation of adequate questions aimed at studying the problem. It is surprising that the most important issue for fundamental science boils down to studying the conditions under which a replicator can indefinitely be subjected to cell selection.

\section{Reference}

[1] Agmon I, Bashan A, Zarivach R, Yonath A (2005) Symmetry at the active site of the ribosome: structural and functional implications. Biol. Chem. 386: 833-844.

[2] Atkins JF, Gesteland RF, Cech TR (2011) RNA Worlds: From Life's Origins to Diversity in Gene Regulation. Cold Spring Harb. Lab. Press, p. 366.

[3] Attwater J, Wochner A, Holliger P (2013) In-ice evolution of RNA polymerase ribozyme activity. Nature Chemistry 5:1011-1018.

[4] Barrick JE, Yu DS, Yoon SH, Jeong H, Oh TK, Schneider D, Lenski RE, Kim JF (2009) Genome Evolution and Adaptation in a Long-Term Experiment with Escherichia coli. Nature 461:12431247.

[5] Belshaw R, Pybus OG, Rambaut A (2007) The evolution of genome compression and genomic novelty in RNA viruses. Genome Res. 17:1496-1504

[6] Bokov K, Steinberg S (2009) A hierarchical model for evolution of 23S ribosomal RNA. Nature 457:977-980.

[7] Briones C, Stitch M, Manrubia SC (2009) The dawn of the RNA World: Toward functional complexity through ligation of random RNA oligomers. RNA 15:743-749.

[8] Cairns-Smith AG (1982) Genetic takeover and the mineral origins of life. Cambridge University Press, p. 488.

[9] Chetverina HV, Demidenko AA, Ugarov VI., Chetverin AB (1999) Spontaneous rearrangements in RNA sequences. FEBS Letters 450:89-94.

[10] Lawrence AD., Alm EJ (2011) Rapid evolutionary innovation during an Archaean genetic expansion. Nature 469:93-96.

[11] Dibrova DV, Makarova KS, Galperin MY, Koonin EV, Mulkidjanian AY (2011) Comparative analysis of lipid biosynthesis in archaea, bacteria and eukaryotes: What was the structure of the first membrane lipids? Proceedings of the International Moscow Conference on Computational Molecular Biology, Moscow State University, Moscow, pp. 92-93.

[12] Doudna JA, Cech TR (2002) The Chemical Repertoire of Natural Ribozymes. Nature 418: 222-228.

[13] Fedor M.J, Williamson JR (2005) The Catalytic Diversity of RNAs. Nature Reviews Molecular Cell Biology 6: 399-412.

[14] Ferris JP, Hill AR, Liu R, Orgel IE (1996) Synthesis of long prebiotic oligomers on mineral surfaces. Nature 381: 59-61.

[15] Freeland SJ, Hurst LD (1998) The Genetic Code Is One in a Million. J Mol Evol 47:238-248.

[16] Gorbalenya AE, Koonin EV (1989) Viral Proteins Containing the Purine NTP-Binding Sequence Pattern. Nucleic Acids Res. 17: 8413-8440.

[17] Ikehara K (2002) Origins of gene, genetic code, protein and life: comprehensive view of life system from a GNC-SNS primitive genetic code hypothesis. J. Biosci 27: 165-186. 
[18] Ikehara K, Omori Y, Arai R, Hirose A (2002) A novel theory on the origin of the genetic code: a GNS-SNS hypothesis. J. Mol. Evol. 54:530-538.

[19] Koonin EV, Martin W (2005) On the Origin of Genomes and Cells within Inorganic Compartments. Trends Genet. 21:647-654.

[20] Koonin EV (2009) On the Origin of Cells and Viruses: Primordial Virus World Scenario. Ann N Y Acad Sci. 1178: 47-64.

[21] Koonin EV (2009) Evolution of Genome Architecture. Int J Biochem Cell Biol. 41:298-306.

[22] Koonin EV (2006) Temporal order of evolution of DNA replication systems inferred by comparison of cellular and viral DNA polymerases. Biol Direct. 1:39.

[23] Koonin EV (2003) Comparative Genomics, minimal gene-sets and the Last Universal Common Ancestor. Nat. Rev. Microbiol. 1:127136.

[24] Levitt M. (2009) Nature of the Protein Universe. Proc. Natl. Acad. Sci. USA 106: 11079-11084.

[25] Leipe D, Aravind L, Koonin E (1999) Did DNA Replication Evolve Twice Independently? Nucleic Acids Res. 27:3389-3401.

[26] Lincoln TA, Joyce GF (2009) Self-sustained replication of an RNA enzyme. Science 323:1229-1232.

[27] Martin W, Russell MJ (2003) On the Origins of Cells: A Hypothesis for the Evolutionary Transitions from Abiotic Geochemistry to Chemoautotrophic Prokaryotes, and from Prokaryotes to Nucleated Cells. Philos Trans R Soc Lond B Biol Sci. 358: 59-83

[28] Miller SL (1986) Current status of the prebiotic synthesis of small molecules. Chem. Scr. 26B: 5-11.

[29] Mulkidjanian AY (2009) On the origin of life in the Zinc world, Validation of the hypothesis on the photosynthesizing zinc sulfide edifices as cradles of life on Earth. Biol Direct. 4:27.

[30] Mulkidjanian AY, Makarova KS, Galperin MY., Koonin EV (2007) Inventing the Dynamo Machine: The Evolution of the F-type and V-type ATPases. Nature Reviews Microbiology 5: 892-899.

[31] Mulkidjanian AY, Galperin MY, Makarova KS, Wolf YI, Koonin EV (2008) Evolutionary primacy of sodium bioenergetics. Biol Direc. 3:13.

[32] Mulkidjanian AY, Dibrov P, Galperin MY (2008) The past and present of sodium energetics: May the sodiummotive force be with you. Biochimica et Biophysica Acta (BBA) -Bioenergetics 1777. I. 7: P. 985-992.

[33] Mulkidjanian AY, Bychkov AY, Dibrova DV, Galperin MY, Koonin EV (2012) Origin of first cells at terrestrial, anoxic geothermal fields. Proc Natl Acad Sci USA Feb 13: 821-830.

[34] Nissen P, Hansen J, Ban N, Moore PB, Steitz TA (2000) The structural basis of ribosome activity in peptide bond synthesis. Science 289:920-930

[35] Nisbet EG, Fowler. CM (1996) Some liked it hot. Nature 382: 404 405.

[36] Novozhilov AS, Koonin EV (2009) Exceptional Error Minimization in Putative Primordial Genetic Codes. Biol Direct. 4: 44

[37] Oba T, Fukushima J, Maruyama M, Iwamoto R, Ikehara K (2005) Catalytic activities of $\square$ GADV $\square$-petides. Ori. Life Evol. Bioshph. 35: 447-460.

[38] Omelchenko MV, Galperin MY, Wolf YI, Koonin EV (2010) NonHomologous Isofunctional Enzymes: A Systematic Analysis of Alternative Solutions in Enzyme Evolution. Biol Direc. 5:31.

[39] Orgel LE (1992) Molecular replication and the origin of life. Nature 358: 203-209.

[40] Pereto J, Lopez-Garcia P, Moreira D (2004) Ancestral Lipid Biosynthesis and Early Membrane Evolution. Trends Biochem Sci. 29:469-477.

[41] Poole A, Penny D, Sjoberg B-M (2000) Methyl-RNA: an evolutionary bridge between RNA and DNA? Chem Biol. 7: 207-216.

[42] Root-Bernstein M, Root-Bernstein R (20015) The ribosome as a missing link in the evolution of life. J. Theor. Biol. 367: 130-158.

[43] Russell MJ, Martin W (2004) The rocky roots of the acetil-CoA pathway.Trends Biochem. Sci. 29:358-363.

[44] Russell MJ, Hall AJ, Cairns-Smith AG, Braterman PS (1988) Submarine hot springs and the origin of life. Nature 336:117.

[45] Russell MJ (2006) First life. American Scientist 94:32-39.

[46] Stetter KO (2006) Hyperthermophiles in the history of life. Phil. Trans. R. Soc. B. 361:1837-1843.

[47] Trifonov EN, Gabdank I, Barash D, Sobolevsky Y (2006) Primordia Vita. Deconvolution from Modern Sequences. Orig Life Evol Biosph. 36:559-565.

[48] Turk RM, Chumachenko NV, Yarus M (2010) Multiple Translational Products from a Five-Nucleotide Ribozyme. Proc. Natl. Acad. Sci. USA 107:4585-4589.
[49] Vaidya N, Manapat ML, Chen IA, Xulvi-Brunet R, Hayden EJ, Lehman N (2012) Spontaneous network formation among cooperative RNA replicators. Nature 491:72-77.

[50] Vetsigian K, Woese C, Goldenfeld N (2006) Collective Evolution and the Genetic Code. Proc Natl Acad Sci USA 103:10696-10701.

[51] Vortler S, Morl M (2010) tRNA-nucleotidyltransferases: Highly unusual RNA polymerases with vital functions. FEBS Letters 584:297-302.

[52] Wachtershauser G (1990) Evolution of the first metabolic cycles. Proc. Natl. Acad. Sci. USA 87:200-204.

[53] Wachtershauser G (2006) From volcanic origins of chemoautotrophic life to Bacteria, Archaea and Eukarya. Phil.Trans. R. Soc. B 361:1787-1808.

[54] Wachtershauser G (1992) Groundworks for an evolutionary biochemistry: the iron-sulfur world. Prog. Biophys. Mol.Biol. 58:185201.

[55] Wolf YI, Brenner SE, Bash PA, Koonin EV (1999) Distribution of Protein Folds in the Three Superkingdoms of Life. Genome Res 9:17-26.

[56] Wolf YI, Koonin EV (2007) On the origin of the translation system and the genetic code in the RNA world by means of natural selection, exaptation, and subfunctionalization. Biology Direct. May 31:2-14.

[57] Wochner A, Attwater J, Coulson A, Holliger P (2011) RibozymeCatalyzed Transcription of an Active Ribozyme. Science 332:209 212.

[58] Wolf YI, Koonin EV (2007) On the origin of the translation system and the genetic code in the RNA world by means of natural selection, exaptation, and subfunctionalization. Biology Direct 2:14.

[59] Woese CR (2002) On the Evolution of Cells. Proc. Natl. Acad. Sci. USA 99:8742-8747.

[60] Yuan J, Palioura S, Salazar JC, Su D, O'Donoghue P, Hohn MJ, Cardoso AM, Whitman WB, Soll D (2006) RNA-dependent conversion of phosphoserine forms selenocysteine in eukaryotes and archaea. Proc. Natl. Acad. Sci. USA 103: 18923-18927. 